\title{
FATRAS - A Novel Fast Track Simulation Engine for the ATLAS Experiment
}

\author{
Sebastian Fleischmann* \\ Physikalisches Institut, University of Bonn, Germany \\ E-mail: Sebastian.Fleischmann@cern.ch
}

\section{on behalf of the ATLAS Collaboration}

\begin{abstract}
Monte Carlo simulation of the detector response is an essential part of any kind of analysis of contemporary High Energy Physics experimental data. At the LHC these simulated data sets are needed with large statistics and high precision level, which makes their production a CPUintensive task. ATLAS has thus concentrated on optimising both full and fast detector simulation techniques to achieve this goal within the computing limits of the collaboration. At the early stages of data-taking, in particular, it is necessary to reprocess Monte Carlo event samples continuously, while tuning the simulation modules to improve the agreement with the data taken from the detector itself.

We present a new, fast track simulation engine which implements a full Monte Carlo simulation based on modules and the geometry of the standard ATLAS track reconstruction application. This is combined with a fast parametric-response simulation of the Calorimeter. This approach shows a high level of agreement with the full simulation, while achieving a relative timing gain of about 100. FATRAS was designed to provide a fast feedback cycle for tuning the MC simulation with real data: this includes the material distribution inside the detector, the integration of misalignment and conditions status, like inactive detector modules, dead and noisy channels, as well as calibration at the hit level. We explain the concepts of the fast track simulation and show the performance with respect to first data taken with the ATLAS detector in December 2009.
\end{abstract}

13th International Workshop on Advanced Computing and Analysis Techniques in Physics Research February 22-27, 2010

Jaipur, India

\footnotetext{
* Speaker.
} 


\section{Introduction}

Monte Carlo simulations of the detector response are essential in High Energy Physics to compare theoretical predictions to measured data. Various Monte Carlo event generators exist to create simulated samples of collision events including shower evolution from the hadronisation of quarks and gluons. However, those final states cannot directly be compared to observables, because detector acceptances and reconstruction efficiencies have to be taken into account. Modern detectors in High Energy Physics are extremely complex systems of sub-detectors of different technologies. Therefore, only Monte Carlo techniques are applicable to include detector effects in the prediction of observables from the generated event samples.

In the ATLAS experiment [1] at the Large Hadron Collider (LHC) the detailed detector simulation is based on the widely used Geant 4 package [2]. Geant 4 simulates particles traversing the ATLAS detector including bending in the magnetic field, interactions with the detector material and particle decays. In a second step, the so-called digitisation, the signal produced in the active detector elements is converted into data objects representing the detector measurements by simulating the response of the detector electronics.

However, the detailed simulation of particles penetrating the detector is a very CPU-time consuming task. For example the simulation of a single $t \bar{t}$ event takes about $30 \mathrm{kSI} 2 \mathrm{Kminutes}$ [3]. Therefore, the ATLAS collaboration decided to adopt a three-fold strategy in its detector simulation. In addition to the full Geant 4-based simulation the fast simulation ATLFAST-I exists, which uses a parametric approach. It directly includes all effects on momentum and energy resolution and reconstruction efficiencies in the parametrisation. High-level objects used in the physics analyses are directly produced from the output of the Monte Carlo event generator. In recent times this has been complemented by ATLFAST-II, which uses a simplified detector model, but still allows to run the full reconstruction chain of the ATLAS software. Hence it is more detailed for example in the simulation of correlations between objects and additional fake tracks. As another advantage no parametrisation has to be modified in case of changes in the reconstruction software.

FATRAS is part of ATLFAST-IIF and simulates tracks of charged particles in the ATLAS Inner Detector and the Muon Spectrometer. It uses the extrapolation engine of the ATLAS reconstruction software [4], while including all important material interactions like multiple scattering, energy loss, bremsstrahlung, photon conversions and hadronic interactions. Measurements are simulated along the path of charged particles using an own implementation of the digitisation.

Section 2 describes the simulation strategy followed by FATRAS in more detail and compares it to the conventional methods. Section 3 shows the performance of FATRAS in comparison to the full Geant 4-based simulation and to collision data at a center of mass energy of $\sqrt{s}=900 \mathrm{GeV}$ recorded by the ATLAS experiment in December 2009.

\section{Strategies for the track simulation in the ATLAS detector}

In recent years the track reconstruction software of the ATLAS experiment was redesigned with modularity and extensibility in mind [5]. Within this effort a need emerged for a quick method to validate different parts of the reconstruction chain from pattern recognition to extrapolation and track fits. During the last years FATRAS evolved from a validation environment to a fully-fledged 
track simulation engine for the ATLAS experiment. This has been achieved by reusing certain modules of the reconstruction software or replacing them with Monte Carlo versions. For example the module to estimate the energy loss of particles penetrating detector layers was supplemented by a version that simulates the energy loss according to the Bethe-Bloch formula and radiation loss according to the Bethe-Heitler formula.

The whole simulation process in FATRAS is based on the so-called Tracking Geometry, which is also used during track reconstruction. The Tracking Geometry is a simplified detector description, that is derived from the detailed geometry model of the ATLAS detector implemented for the Geant 4 simulation. Spatially extended parts of the detector are subsumed in material layers. Especially for the rather thin Pixels and silicon strip modules of the ATLAS detector the layerbased description is a very good approximation. Active detector layers in the tracking system are identical between the full simulation and FATRAS.

FATRAS takes generated events in the HepMC format as an input. In a preparation step a particle stack is filled with all relevant final state particles. By default the primary vertex position of those particles is shifted to the beam spot position as provided by the detector conditions data base and smeared according to its resolution. Arbitrary vertex positions can be simulated as well. Next, all particles of the stack are processed in sequence. For unstable particles the path length up to the decay is simulated. Charged and neutral particles are extrapolated through the detector stepwise from one material layer to the next. In each of these steps detector material effects are simulated. The extrapolation methods range from simple analytic straight line propagations for neutral particles to Runge-Kutta integration methods in the magnetic field for charged particles.

Simulated material effects include bremsstrahlung for electrons, conversion to $e^{+} e^{-}$-pairs for photons, multiple scattering and energy loss ${ }^{1}$. The value of the interaction lengths of the traversed material are estimated from the detector description of the Tracking Geometry. All of the above effects can be estimated from "first principles" like the Bethe-Bloch formula. However, hadronic interactions cannot be simulated that way. FATRAS uses a parametrisation obtained from simulated Geant 4 events instead. Figure 1 shows an example of a single electron event in the ATLAS Inner Detector simulated with FATRAS. The effects of interactions with the detector material are clearly visible here. Secondary particles from interactions are put on FATRAS' particle stack to be further propagated through the detector, until they fall below a certain threshold in their transverse momentum. Particle decays inside the detector are simulated via direct use of the corresponding Geant 4 module to obtain the types and 4-momenta of the decay products. Therefore all decay modes implemented in Geant are available in FATRAS as well.

FATRAS uses a geometrical model to create clusters of activated pixels and silicon strips corresponding to the ionisation created by the particles crossing a detector module of the Pixels or silicon strip (SCT) detector. In this model the path length of the track inside each pixel or strip of the module is calculated. According to that the activated pixels and a simple charge sharing among them are determined. This model leaves only two parameters to be tuned to data, the minimal path length in a pixel to be activated and the smearing of the charge deposition. It also incorporates the effect of the so-called Lorentz angle. Due to the magnetic field in the ATLAS Inner Detector

\footnotetext{
${ }^{1}$ The energy loss due to ionisation is simulated according to a Landau distribution following the Bethe-Bloch theory. Delta rays of secondary electrons are not yet included.
} 


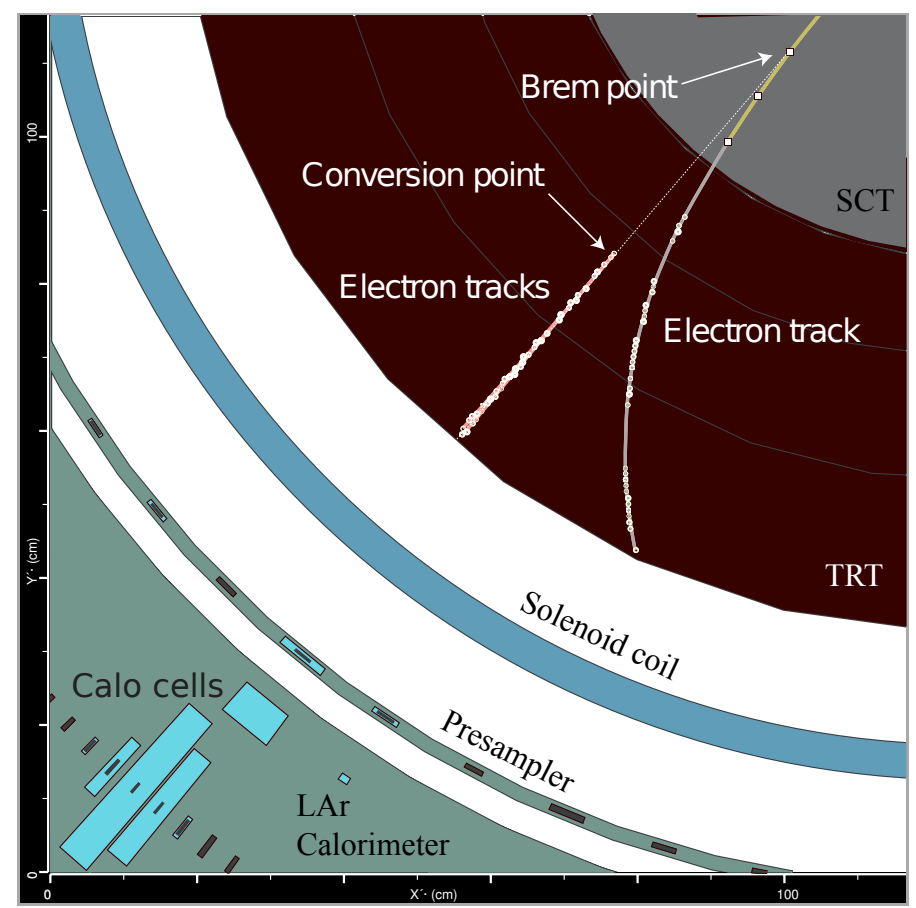

Figure 1: Event display of a single electron event simulated with FATRAS for the Inner Detector and the fast calorimeter simulation FastCaloSim for the calorimeter response. The electron creates a photon by bremsstrahlung in the ATLAS silicon tracker (SCT), which itself converts into an $e^{+} e^{-}$-pair inside the Transition Radiation Tracker (TRT).

not only the particles themselves are deflected, but also the drifting electrons they create inside the silicon by ionisation. The cluster size and position are therefore modified depending on the orientation of the detector module with respect to the magnetic field (compare figure 4(b)). The FATRAS simulation of measurements in the ATLAS Transition Radiation Tracker (TRT) is much simpler. At the moment it is purely based on a smearing of the measurement position. Future plans foresee the adoption of at least part of the TRT digitisation used by the full detector simulation.

After the whole particle stack has been processed, all final particles are extrapolated to the entrance surface of the calorimeter. They are picked up by the subsequent calorimeter simulation. In figure 1 it can be seen how the secondary (or even tertiary) particles created by FATRAS are used for the calorimeter simulation. The example uses the fast calorimeter simulation FastCaloSim [6]. Muons crossing the calorimeters can afterwards be handed back to the Muon System part of FATRAS.

In a last post-processing step measurements are extracted from the simulated tracks. Noise measurements can be added at the level of individual pixels or strips in the silicon detectors. Overlapping clusters are merged, so that FATRAS can provide rather precise predictions of two-track resolutions. In the TRT noise hits will mask measurements from particles.

FATRAS is very modular, which allows to easily add more effects in the simulation, when discrepancies between FATRAS and full simulation or data are observed. As already mentioned a big advantage compared to conventional fast simulations techniques is the full compatibility of the 
output to full simulation and data and the ability to run the standard reconstruction algorithms on it. Especially complex reconstructed objects profit from this approach. However, certain limitations exist and corrections at the level of objects used for the physics analyses are needed. ATLAS provides a general framework for such corrections.

Besides being fast FATRAS also allows to perform studies, that are difficult to do with the full simulation only. For example detector geometries can easily be exchanged. FATRAS was therefore used to compare various potential layouts of a new Inner Detector for the ATLAS experiment as it will be needed for high luminosity upgrades of the LHC. FATRAS allowed to give precise estimates of detector occupancies and other important factors depending on the luminosity. Only layouts that had been proven to be promising using FATRAS needed to be simulated based on Geant 4 .

\section{Performance of FATRAS}

FATRAS was validated mainly by comparing it to the full, Geant 4 based simulation of the ATLAS detector. Starting from the validation of basic track properties in single particle samples, more and more complex reconstructed objects like hadronic decays of tau leptons were compared and tuned. In nearly all quantities a good agreement could be achieved. Remaining discrepancies are under investigation and mostly understood. All comparisons were made in terms of their relevance for observables accessible by the final reconstruction. For example, the composition of secondaries from hadronic interactions are assumed to be of lesser importance as those secondaries cannot be reconstructed anyhow.

Figure 2 shows the momentum distribution of hard photons that are emitted from simulated electrons and the residual of the transverse impact parameter $d_{0}$ for muon tracks, respectively. It is clearly visible in figure 2(b), that FATRAS performs better than ATLFAST-I in the correct description of the tail distributions.

In December 2009 the ATLAS experiment was able to record the first proton-proton collisions in the LHC at a center of mass energy of $\sqrt{s}=900 \mathrm{GeV}$. These minimum bias events allowed us to compare the fast track simulation for the first time with real data from collisions. The comparison concentrated on a single "run", because only in a limited period of time all tracking detectors operated at nominal settings. Still a sufficiently large data sample could be obtained to compare basic tracking quantities between data and simulation.

Figure 3(a) shows the mean number of measurements of the Pixels detector associated to reconstructed tracks versus the pseudorapidity $\eta^{2}$. The sinusoidal-like shape in the central part of the distribution comes from a convolution of two effects: First, two detector modules in the same layer have been inactive during the run, which means that tracks in this region hit only two instead of three modules. Secondly, the beam spot position along the beam (z-) axis was shifted a few centimeters with respect to the nominal center of the detector, which leads to a shift between the $\eta$ angle at the beam spot position and the $\eta$ angle of the position of the inactive detector modules. Figure 3(b) shows the same for the azimuthal angle $\phi$. The good agreement between simulation and data shows, that the fast track simulation does not only describe the detector geometry correctly, but also includes information about the detector conditions changing from run to run. FATRAS

\footnotetext{
${ }^{2} \eta=-\ln \tan (\theta / 2)$, where $\theta$ is the polar angle relative to the beam axis
} 


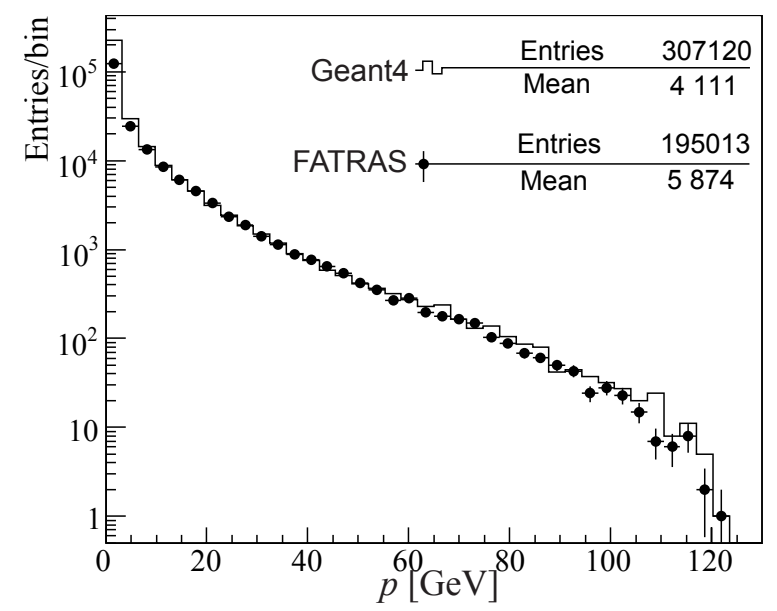

(a) Momentum distribution of brem photons

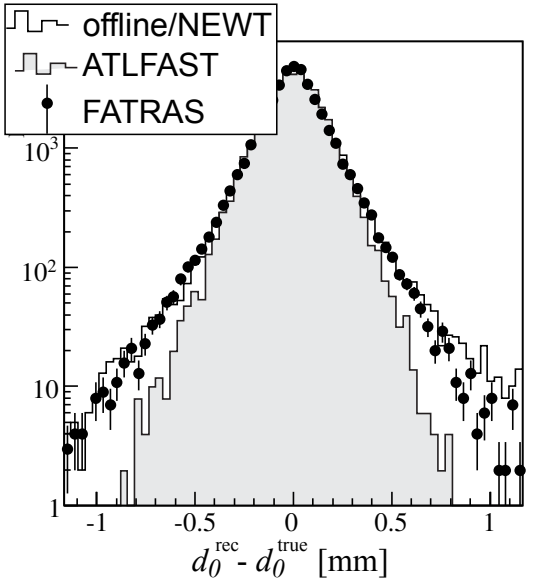

(b) Residual of the transverse impact parameter $d_{0}$

Figure 2: Comparison of FATRAS to full the Geant 4 simulation: (a) shows the momentum distribution of hard photons that are emitted from simulated electrons with transverse momenta of $p_{\mathrm{T}}=15 \mathrm{GeV}$ restricted to the pseudo rapidity range $|\eta|<2.5$. Discrepancies in the very low momentum region are due to a cut-off in the photon processing in FATRAS. (b) shows the residual of the the transverse impact parameter $d_{0}$ for single muon tracks in the low momentum limit $\left(p_{\mathrm{T}}=1 \mathrm{GeV}\right)$ over the entire acceptance range of $|\eta|<2.5$ of the Inner Detector. Results obtained from the full simulation (solid line) are compared to FATRAS tracks (dots) and results from the parametrisation-based ATLFAST-I simulation (shaded area).

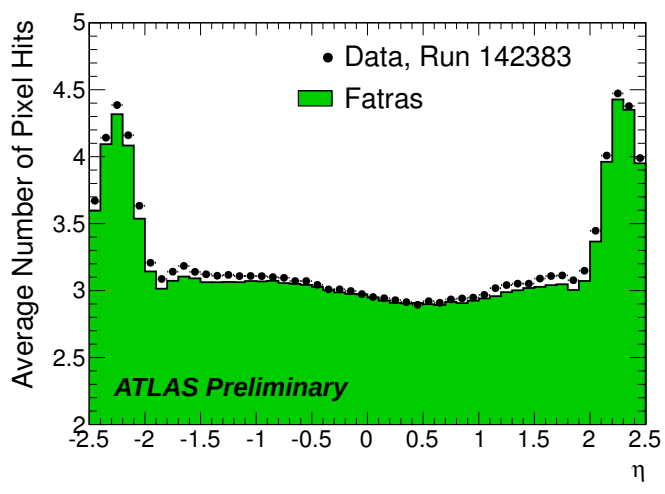

(a) As a function of $\eta$

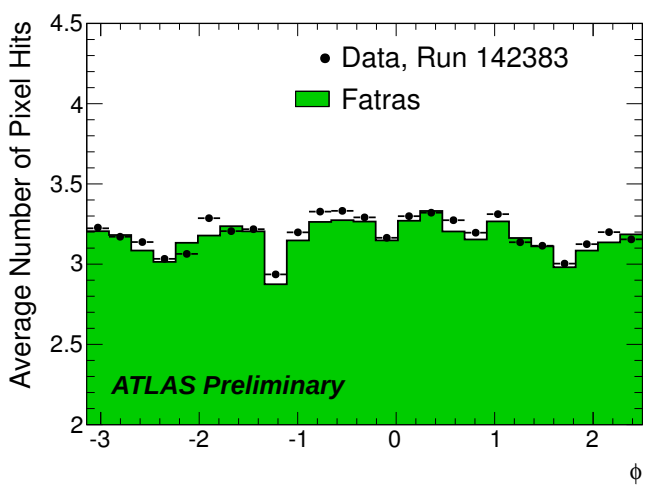

(b) As a function of $\phi$

Figure 3: Average number of pixel hits per selected track as a function of pseudorapidity $\eta$ and azimuthal angle $\phi$ of the track, respectively. Comparison of the FATRAS Monte Carlo and the data is shown. The structure is mainly determined by the inactive pixel modules that have been also masked in the digitisation process of the MC samples to reproduce the run conditions.

automatically includes conditions data like the beam spot position and size and inactive or masked detector modules.

The clusterisation model of FATRAS was also tested with the first data. As illustrated in 
figure 4(b) the cluster size depends on the track incident angle on the detector module. Figure 4(a) shows the measured and simulated mean cluster size versus the track incident angle in the Pixels detector. FATRAS has not been tuned to data yet, but already now a reasonably good agreement could be achieved.

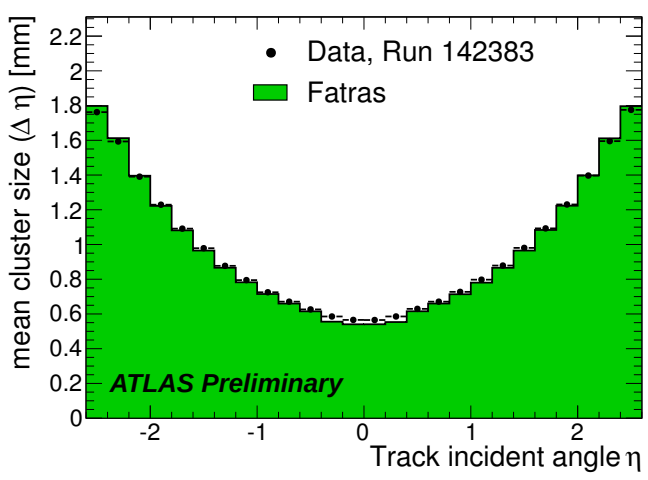

(a) Mean cluster size in the Pixels detector versus track incident angle $\eta$ for good tracks comparing FATRAS simulation with data.

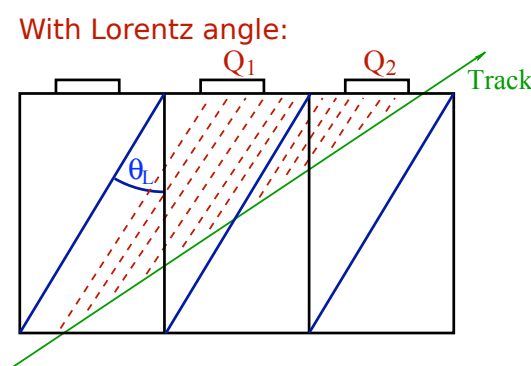

(b) Sketch of the clusterisation model in FATRAS illustrating the dependency of the cluster size on the incident angle of the track with respect to the detector module.

Figure 4: Comparison of the FATRAS geometrical clusterisation model in the Pixels detector with the data.

The typical time to simulate a $t \bar{t}$ event in the whole ATLAS detector reduces from about $2000 \mathrm{kSI} 2 \mathrm{Kseconds}$ for full Geant 4 to $\approx 100 \mathrm{kSI} 2 \mathrm{Kseconds}$ when using the fast calorimeter simulation FastCaloSim, but keeping Geant 4 for Inner Detector and Muon System. One gains another factor of more than 10 , when using FATRAS for the track simulation $(\approx 7 \mathrm{kSI} 2 \mathrm{Kseconds})$ [3]. The simulation time of the Inner Detector reduces from $\approx 146 \mathrm{kSI} 2 \mathrm{Kseconds}$ (simulation) + $4.3 \mathrm{kSI} 2 \mathrm{Kseconds}$ (digitisation) for Geant 4 to $\approx 2.8 \mathrm{kSI} 2 \mathrm{Kseconds}$ (total) for FATRAS compared to about $0.02 \mathrm{kSI} 2 \mathrm{Kseconds}$ for the parametrisation based simulation ATLfast-I [7]. A further speed-up of FATRAS would not improve the overall timing as the FATRAS simulation takes already about the same amount of CPU-time as the Inner Detector reconstruction chain.

\section{Conclusion}

We presented the basic concepts of our new fast approach for track simulation in the ATLAS experiment. FATRAS evolved to a fully-fledged track simulation engine including the most important effects of material interactions and read-out electronics. Contrary to previous fast simulations it provides detector simulation at the hit level and allows to run the full track reconstruction chain. FATRAS will neither replace the full simulation nor the very fast simulation ATLfast-I, but it complements the two with new applications. The simulation time of the ATLAS Inner Detector can be reduced by a factor of 50 in typical $t \bar{t}$ events compared to the full Geant 4 based simulation. Comparisons of FATRAS to the full simulation and to first collisions data showed a good agreement. 


\section{References}

[1] The ATLAS Collaboration, The Atlas Experiment at the CERN Large Hadron Collider, 2008 JINST 3 S08003.

[2] S. Agostinelli et al., Geant4 - a simulation toolkit, Nucl. Instr. Methods Phys. Res. A 506 (2003) 250-303.

[3] The ATLAS Collaboration, The ATLAS Simulation Infrastructure, to be submitted to EPJC (available as ATLAS Internal Note ATL-SOFT-INT-2010-002)

[4] A. Salzburger, The ATLAS Extrapolation package, ATLAS Note, ATL-SOFT-PUB-2007-005, 2007.

[5] A. Salzburger (Editor) et al., Concepts, Design and Implementation of the ATLAS New Tracking (NEWT), ATLAS Note, ATL-SOFT-PUB-2007-002, 2007.

[6] M. Dührssen, The fast calorimeter simulation FastCaloSim, ATLAS Internal Note, ATL-PHYS-INT-2008-043, 2008

[7] K. Edmonds et al., The Fast ATLAS Track Simulation (FATRAS), ATLAS Note, ATL-SOFT-PUB-2008-002, 2008. 